\title{
El Subphylum Mucoromycotina: generalidades y aspectos taxonómicos recientes
}

\author{
(Mucoromycotina Subphylum: overview and recent taxonomic aspects)
}

Recibido:7-06-2013

Aprobado:11-06-2013

Palabras clave: mucorales, Mucoromycotina, zygomycota.

Key words: mucorales, Mucoromycotina, zygomycota.

\section{RESUMEN}

El subphylum Mucoromycotina es un grupo de hongos ubicuos, saprobios, caracterizados por la presencia de hifas cenocíticas. También se caracterizan por presentar una fase sexual con la formación de una zigospora. Los mucormycetes son capaces de infectar plantas, animales y seres humanos. Recientemente, varios estudios han comunicado de la creciente incidencia de mucormicosis en pacientes inmunocomprometidos e inmunocompetentes. Dados los avances en biología molecular, la sistemática de este grupo de hongos ha experimentado varios cambios taxonómicos en los últimos años. Este artículo discutirá los principales cambios taxonómicos, en especial de los géneros y especies de interés clínico.

\section{Características generales de los mucormycetes}

Los mucormycetes representan aproximadamente el $1 \%$ de las especies (poco más de unas 1000) de los hongos (reino Fungi) descritas hasta el presente (Kirk et al, 2008). Debido a que se estima que el número mínimo de hongos rondaría el millón y medio de especies (Hawksworth, 1991, 2001; Kirk et al., 2001; 2008), el número total de mucormycetes todavía por describir sería de, al menos, unos 10.000. (Hawksworth, 1991; Hawksworth y Rossman, 1997; Hawksworth, 2001; Kirk et al, 2001; 2008). Según algunos autores, los mucormycetes serían los primeros organismos que colonizaron la tierra, incluso antes que las plantas, hace 600-1.400 millones de años (Berbee y Taylor 2001; Heckman et al., 2001).
Eduardo Álvarez Duarte.

nidad Micología - Hongos Filamentosos, Programa Microbiología ía, Instituto Ciencias Biomédicas, Universidad de Chile. Autor para correspondencia: ealvarezd@med.uchile.cl 
El Subphylum Mucoromycotina: generalidades y aspectos taxonómicos recientes - E. Alvarez.

economía, causando pudrición durante el almacenamiento de los vegetales y carne. Otras especies como Apophysomyces elegans, Mucor circinelloides, Rhizopus oryzae y Saksenaea vasiformis, son capaces de infectar tanto las personas inmunocomprometidas como inmunocompetentes (de Hoog et al, 2000; Ribes et al, 2000; Roden et al, 2005). Algunas de estas especies son comúnmente aisladas de animales y suelo de zonas tropicales y subtropicales.

Los mucormycetes se caracterizan por su rápido crecimiento en sustratos con alto contenido de azúcar, siendo también comunes en los ecosistemas terrestres y acuáticos, presentando una distribución cosmopolita. Macroscópicamente, muestran un micelio en expansión con un aspecto lanoso, principalmente blanco-gris (raramente naranja 0 marrón), que puede alcanzar varios centímetros en altura, como en las especies Blakeslea y Phycomyces. Microscópicamente, se caracterizan por hifas de paredes delgadas, compuestas principalmente por quitina y glucanos, presentando generalmente ramificaciones en ángulos rectos. A diferencia de los miembros de los Phylum Ascomycota y Basidiomycota, cuyas hifas están regularmente septadas, la mayor parte de los mucormycetes por lo general presentan micelio cenocítico. Un carácter único que define a los mucormycetes es la producción de esporas sexuales llamados zigosporas, que son generalmente estructuras pigmentadas con una pared de superficie lisa o rugosa, formadas por la fusión de hifas especializada (gametangios) durante la fase sexual (Benjamin, 1979; Benny et al, 2001). Las zygosporas tienen la capacidad de permanecer en estado latente durante un largo período de tiempo, pudiendo germinar cuando existan condiciones ambientales favorables, generando un nuevo ciclo de vida.

La reproducción asexual de los mucormycetes se basa en la producción de esporangiosporas, las cuales pueden estar contenidas en esporangios, dispuestos sobre el extremo de una hifa fértil (esporangióforo). Las esporangiosporas se distinguen de otros tipos de esporas asexuales, como los conidios de Ascomycota y Basidiomycota, por su desarrollo: se forman por la división interna del citoplasma al interior del esporangio. Los esporangios pueden producir desde varias decenas a varios cientos de esporangiosporas. En la madurez, la pared del esporangio normalmente se desintegra, liberando así las esporas, que son generalmente dispersadas por el viento, el agua 0 animales (especialmente artrópodos) (Richardson, 2009). Los esporangios incluyen dos variedades morfológicas: los esporangíolos y los merosporangios. Los esporangíolos son simplemente esporangios monosporados, o contenedores de unas pocas esporas (hasta 30). Los merosporangios son esporangios alargados con esporas uniseriadas, dispuestas en cadena, generalmente producidos sobre la superficie de una vesícula o del talo. Una estructura que puede estar presente al interior de un esporangio es la columela, la cual presenta características morfológicas de importancia taxonómica. En condiciones ambientales adversas, los individuos pueden generar, además, clamidosporas, artrosporas y/o blastosporas (Benny, 1995). Otras estructuras vegetativas, usualmente presentes en varios géneros y que son taxonómicamente importantes son los rizoides (estructuras en forma de raíz) y los estolones (Kirk et al, 2008).

\section{Epidemiología de las mucormicosis}

El espectro actual los agentes etiológicos de las mucormicosis es más amplio y, sin lugar a dudas, comprende un escenario nuevo, muy distinto al de hace unos pocos de años (Meis y Chakrabarti, 2009).

Diversas publicaciones (Alvarez et al., 2009; Ribes et al., 2000; Roden et al., 2005), demuestran que las especies más frecuentemente aisladas en casos de mucormicosis son Rhizopus oryzae, (comprendiendo casi la mitad de los aislamientos), seguido de $\boldsymbol{R}$. microsporus, Mucor circinelloides, Lichtheimia (Absidia) corymbifera, Rhizomucor pusillus, Cunninghamella bertholletiae, Mucor indicus, Cunninghamella echinulata, y Apophysomyces elegans.

Se estima que la principal vía de entrada de los Mucorales es mediante la inhalación de aerosoles conteniendo esporangiosporas u otros propágulos de dispersión. Algunos estudios experimentales han demostrado que, tras la inhalación de esporas, los hospederos desarrollaron enfermedades del tracto respiratorio superior e inferior, con la posterior diseminación al sistema nervioso central (Reinhardt et al., 1981; Waldorf y Diamond, 1984; Kamei, 2001). En este sentido, han sido observados brotes de mucormicosis rinocerebral y pulmonar en ambientes laborales en que se formaban gran cantidad de aerosoles, tales como en excavaciones, construcciones o reformas de edificios (England et al., 1981; 
El Subphylum Mucoromycotina: generalidades y aspectos taxonómicos recientes - E. Alvarez.

Krasinski et al., 1985).

Otra vía de entrada para la instauración de una infección es la cutánea. En términos generales, se conoce la nula capacidad de penetración transcutánea espontánea por parte de los Mucorales. Sin embargo, procesos tales como quemaduras, laceraciones, maceraciones de la piel (Ledgard et al., 2008; Stewardson et al., 2009; Vega et al., 2006), picaduras de insectos o pájaros (Lechevalier et al., 2008; SaraviaFlores et al., 2010; Wilson, 2008), inoculación traumática (Andresen et al., 2005; Ribes et al., 2000; Snell y Tavakoli, 2007), y cualquier proceso de alteración de esta barrera permiten la entrada y la colonización de los tejidos por parte de dichos microorganismos.

La vía oral de entrada (debido a la ingestión de bebidas 0 alimentos contaminados con esporas) también ha sido involucrada en la producción de infecciones, aunque su incidencia es relativamente baja en humanos, pudiendo jugar un papel más importante en la infección de otros animales (Oliver et al., 1996; Richardson, 2009; Sandford et al., 1985).

Las mucormicosis han sido consideradas desde siempre un proceso infeccioso típico de pacientes con inmunodeficiencias. Durante las últimas tres décadas se ha observado el aumento de la incidencia de mucormicosis debido a factores que afectan el normal funcionamiento del sistema inmunitario, tales como el incremento en la incidencia del cáncer, tratamientos inmunosupresores y el uso masivo de corticoesteroides. A pesar que las infecciones por levaduras del género Candida (candidiasis) y por hongos filamentosos del género Aspergillus (aspergilosis) continúan siendo las prevalentes en pacientes inmunocomprometidos, las mucormicosis (zygomicosis) han ido incrementado dramáticamente su incidencia. En Francia, un estudio retrospectivo (1997 - 2006) estableció que el aumento en el número de casos reportados fue de alrededor de 40\% (Bitar et al., 2009). Otro estudio, basado en el número de reportes realizados en artículos en lengua inglesa, estableció que el número de casos entre 1940 y el año 2000 aumentó en un 70\% (Roden et al., 2005). Asimismo, Saegeman y colaboradores (2010), en un estudio realizado en la ciudad de Lovaina (Bélgica), comunicaron un incremento significativo en la incidencia anual de mucormicosis (80\%) entre los años 2000 y 2009.

Durante la última década también se ha observado el aumento de la incidencia de mucormicosis en pacientes inmunocompetentes, principalmente debido a Apophysomyces elegans y Saksenaea vasiformis (Baradkar y Kumar, 2009; Stewardson et al., 2009; Meis y Chakrabarti, 2009). En el caso de Apophysomyces elegans, los cuadros han sido reportados principalmente en áreas tropicales y subtropicales, destacando la India, país que concentra más del $50 \%$ de los casos anuales (Chakrabarti et al., 2003, 2006, 2009; Devi et al., 2008; Jain et al., 2006; Meis y Chakrabarti, 2009; Ribes et al., 2000).

\section{Taxonomía y filogenia}

Tradicionalmente, la clasificación de los mucormycetes se ha basado en caracteres fenotípicos, principalmente en las características morfológicas de las estructuras reproductivas de las fases sexual y asexual, así como también en la presencia y características de ciertas estructuras vegetativas diferenciadas, tales como estolones y rizoides (Benny, 1995; Hoffmann et al., 2009). Varios autores han informado de la importancia taxonómica de la zygospora en la identificación / clasificación de este grupo de hongos (Benjamin, 1979; Weitzman et al, 1995). El apareamiento sexual (según sea heterotálico u homotálico), la ornamentación de las zyposporas y las células suspensorias (hifas que sostienen los gametangios) se han utilizado para resolver algunas controversias taxonómicas (Hoffmann et al., 2007), tales como en Absidia / Mycocladus / Lichtheimia (Hoffmann et al., 2007, 2009). Asimismo, algunos estudios han demostrado la utilidad de la forma y tamaño de las esporangiosporas en la clasificación taxonómica de los mucormycetes (García-Hermoso et al., 2009; Hoffmann et al., 2007). De este modo, la división Zygomycota agrupaba a todos los hongos con micelio cenocítico, y cuya reproducción sexual implicaba la producción de zigosporas, estando dividido en las clases Zygomycetes y Trichomycetes. Los Trichomycetes agrupaban a organismos simbiontes de artrópodos, no patógenos para el ser humano (Alexopoulos et al, 1996), mientras que los Zygomycetes agrupaban, entre otros, organismos patógenos para el ser humano y otros animales (Kwon-Chung y Bennet, 1992). Los Zygomycetes se subdividían en 9 ordenes: Asellariales, Dimargaritales, Endogonales, Entomophthorales, Harpellales, Kickxellales, Mortierellales, Mucorales y Zoopagales (Benjamín, 1979; Benny, 1982; White 
El Subphylum Mucoromycotina: generalidades y aspectos taxonómicos recientes - E. Alvarez.

et al, 2006), siendo los Mucorales y Entomophtorales los de importancia en clínica humana. Asimismo, el orden Mucorales se dividía en 6 familias: Mucoraceae, Cunninghamellaceae, Saksenaceae, Thamnidiaceae, Syncephalastraceae, y Mortierellaceae. Posteriormente, y basado en los estudios llevados a cabo por von Arx (1983), se adicionó la familia Absidiaceae. Benny (1995) señaló las limitaciones de las características morfológicas en la delimitación de especies dentro de ciertos mucormycetes, sugiriendo el uso de herramientas moleculares para la resolución de las diferentes controversias taxonómicas existentes. Otros autores, tales como 0'Donnell y colaboradores (1994) sugirieron la artificialidad del sistema de clasificación tradicional de los mucormycetes, el cual no reflejaba la relaciones filogenéticas entre los taxones. Una serie de estudios moleculares llevados a cabo en los últimos años han demostrado el origen polifilético de los mucorales, confirmándose la hipótesis de la artificial clasificación taxonómica existente (Hibbet et al, 2007; James et al, 2006; Tanabe et al, 2004, 2005; 0'Donnell et al. 2001; Voigt et al, 1999; White et al, 2006). Según estos estudios, solo los phylum Ascomycota y Basidiomycota estaríam bien soportados a nivel filogenético, estableciéndose claramentese su origen monofilético (Berbee y Taylor. 2001; Hibbet et al, 2007). Los cambios mas importantes a nivel taxonómico ocurrieron en el grupo de los antiguamente denominados Zygomycota. Segun esta propuesta, el grupo polifiético Zygomycota fue disuelto en los grupos monofiléticos que se lograron observar como resultafo fe los estudios genéticos. Así, fueron propuestas los subphyla (=subdivisiones) tales como Entomophthoromycotina, Kickxellomycotina, Mucoromycotina y Zoopagomycotina (Hibbet el al., 2007). Así, Ios órdenes Zoopagales y Entomophthorales se asignaron a las subdivisiones Zoopagomycotina y Entomophthoromycotina, respectivamente; Ios Kickxellales, Dimargaritales, Harpellales y Asellariales, al subphylum Kickxellomycotina, y los Mucorales, Endogonales y Mortierellales al subphylum Mucoromycotina, el que agrupa un total de 61 géneros y unas 325 especies (Hibbet et al, 2007; Kirk et al, 2008). (Figuras 1). Recientemente, y dadas las relaciones filogenéticas demostradas; se propuso la reclasificación de los miembros del subphylum Entomophthoromycotina en el nuevo phylum Entomophthoromycota, el cual estaría conformado por las clases Basidiobolomycetes (conformado por Basidiobolales y Basidiobolaceae), Entomophthoromycetes (conformado por Entomophthorales, y restringido a Ancylistaceae, Completoriaceae, Entomophthpraceae y Meristacraceae), y Neogitomycetes (formado por el nuevo orden Neozygitales). Los géneros Ballocephala y Zygemomyces fueron reubicados en el subphylum Kickxellomycotina dado sus características morfológicas coincidentes (Gringaskyi et al., 2012; Humber, 2012).

Actualmente, y según lo anteriormente expuesto, los términos Zigomicetos, Zygomycotina o Zygomycota no son válidos taxonómicamente, por lo cual se sugiere no continuar con su uso. 
El Subphylum Mucoromycotina: generalidades y aspectos taxonómicos recientes - E. Alvarez.

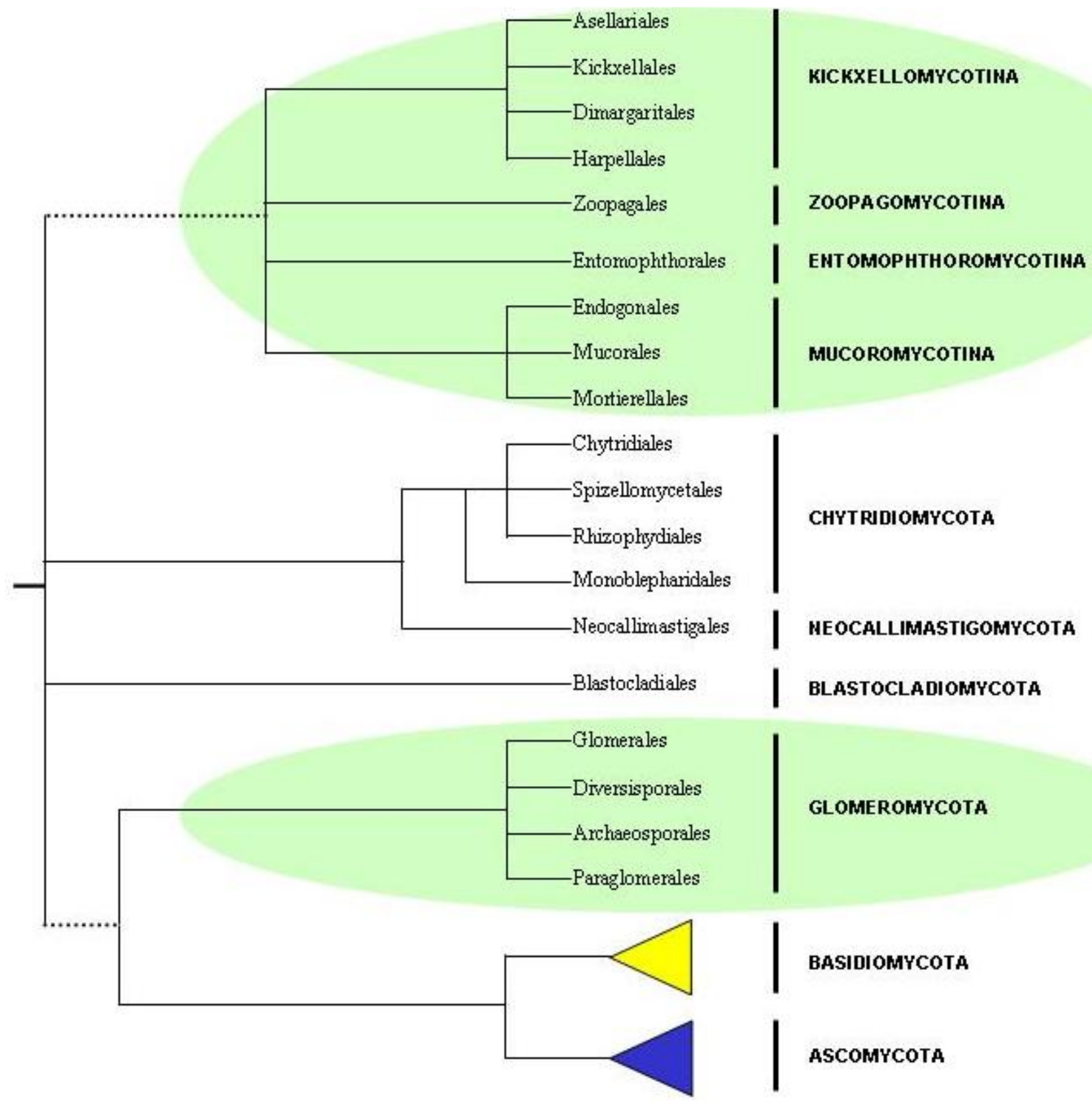

Figura 1. Filogenia molecular y clasificación de los hongos. En color verde los miembros de la antigua división Zygomycota. Con líneas punteadas las relaciones genéticamente inciertas (incertae sedis). Adaptado de Hibbet et al., (2007). 
El Subphylum Mucoromycotina: generalidades y aspectos taxonómicos recientes - E. Alvarez.

\section{Nuevos agentes etiológicos de mucormicosis}

En los últimos años ha sido comunicado por diversos autores el incremento en la incidencia de las mucormicosis a nivel mundial. Asimismo, el espectro de agentes etiológicos ha aumentado, reportándose especies no vinculadas anteriormente en mucormicosis, como también han sido descritas nuevas especies de Mucorales, resolviéndose algunos complejos de especies. Por otro lado, diversos autores comunican el cambio epidemiológico observado; dado los crecientes reportes de mucormicosis en pacientes inmunocompetentes.

Un cambio interesante es lo sucedido en el género Absidia; el cual fue subdividido de acuerdo a sus características fisiológicas, como es la capacidad de crecimiento a diferentes temperaturas. En Absidia s.s fueron agrupadas las especies mesófilas, que no crecen a $37^{\circ} \mathrm{C}$. Por otro lado, las especies no que fueron capaces de crecer a $30^{\circ} \mathrm{C}$ fueron asiganadas al género Lentamyces. Asimismo, las especies capaces de crecer a $37^{\circ} \mathrm{C}$, y por ende, de importancia clínica fueron ubicadas en el género Lichtheimia. De ésta forma, el género Lichtheimia quedó conformado por las especies L. corymbifera, L. blakesleeana y L. hyalospora (Hoffmann et al., 2009). A estos tres miembros, en los últimos años se han añadido nuevas especies tales como L. sphareocystis, L. ornata; y la revalidada L. ramosa (Alastruey-Izquierdo et al., 2010; Garcia-Hermoso et al., 2009).

Mucor es el segundo genero más prevalente en mucormicosis. Históricamente solo 5 especies habían sido descritas en infecciones en seres humanos. Sin embargo, recientemente se han descrito nuevas especies asociadas a mucormicosis. Dichas nuevas especies propuestas corresponden a Mucor ellipsoideus y Mucor velutinosus (Alvarez et al., 2011). Asimismo, se propuso la reubicación de Rhizomucor variabilis var. regularis en el género Mucor, dado las incongruencias existentes entre dicha especie y la descripción de genero existente para Rhizomucor. De este modo, $R$ h. variabilis var. variabilis es conocido hoy en día como Mucor irregularis (Alvarez et al., 2011). Interesante resulta la gran incidencia de $M$. irregularis en mucormicosis, siendo reportado en una serie de casos alrededor del mundo (Hemashettar et al., 2011; Li y Lun., 2012; Schell et al., 2011).

Apophysomyces fue clásicamente conocido como un genero monoespecífico reportado por Misra en el año 1979; siendo reportado principalmente en mucormicosis cutáneas. Dado los análisis genéticos y morfofisiológicos efectuados; fueron descritas 3 nuevas especies; $\boldsymbol{A}$. variabilis, $\boldsymbol{A}$. trapeziformis y $\boldsymbol{A}$. ossiformis (Alvarez et al., 2010a). Según algunos autores; Apophysomyces ha sido considerado un patógeno emergente, destacándose su capacidad de producir infecciones en inmunocompetentes. Por otro lado, resulta interesante la comunicación de 13 casos de mucormicosis cutánea por una de estas nuevas especies descritas luego del tornado Joplin, que afecto a los Estados Unidos en el año 2011 (Neblett et al., 2011).

Saksenaea es un género descrito en el año 1953 en la India; siendo reconocida hasta hace poco solo una única especie, Saksenaea vasiformis. No obstante, recientemente y en base a un estudio polifásico fueron propuestas dos nuevas especies, Saksenaea erythrospora y Saksenaea oblongispora (Alvarez et al., 2010b). Asimismo, Saksenaea vasiformis fue descrito como un complejo de especies, dado la gran variabilidad intraspecifica observada (Alvarez et al., 2010b). Saksenaea también es considerado un patógeno emergente, describiéndose una serie de alrededor del mundo (Taj-Aldeen et al., 2012; Mayayo et al., 2013; Kaushik et al., 2012; Hospenthal et al., 2011; Kompoti et al., 2011)

Muchos mucorales son patógenos oportunistas que causan infecciones diversas, cuyo tratamiento requiere un rápido y fiable diagnóstico (Voigt et al., 1999). El aislamiento en cultivo de estos hongos demanda cierto tiempo y mucho trabajo. La identificación morfológica se ve afectada por el crecimiento atípico de hifas en el tejido humano en comparación con su crecimiento en medios artificiales. Por otra parte, las condiciones óptimas de crecimiento para una fiable identificación morfológica son a menudo desconocidas (Benny, de 1995; Schipper, 1973; Weitzman et al., 1995). En este sentido, aspectos tales como la clasificación propuesta por Hibbett y colaboradores (2007), el acceso a cepas tipo a través de colecciones (CBS, ATCC, ARS, etc.) así como el comparar con secuencias fiables correspondientes a cepas tipo como también de referencia disponibles en bases de datos públicas (GenBank, EMBL, etc.) son importantes requisitos para la identificación molecular fiable.

En En Chile se han reportado una serie de casos hasta la fecha; no obstante, la identificación a nivel especie sigue siendo una tarea pendiente. Especies 
El Subphylum Mucoromycotina: generalidades y aspectos taxonómicos recientes - E. Alvarez.

de Rhizopus y Mucor son las más descritas, concordando estos hallazgos a nivel genérico con lo descrito a nivel mundial (Aguad et al., 2004; Chávez y Rahal., 2004; Cruz y Piontelli, 2011; Oddo y Acuña, 1988; Spalloni et al., 2004; Täger et al., 2012; Thompson et al., 1987; Villarroel, 1985).

\section{REFERENCIAS}

Aguad L, Quezada M, Rahal M, Vallejos M, et al., (2004) Mucormicosis rinosinusal en un paciente infectado por VIH. Rev Chil Infectol 2004; 21: 345-50.

Alastruey-Izquierdo A., Hoffmann K., de Hoog G.S., et al., (2010). Species recognition and clinical relevance of the zygomycetous genus Lichtheimia (syn. Absidia pro parte, Mycocladus). J Clin Microbiol 48: 2154-2170.

Alexopoulos, C.J.; Mims C.W. \& Blackwell M. (1996). Introductory mycology. John Wiley and Sons, New York.

Alvarez, E., Sutton D.A., et al., (2009). Spectrum of zygomycete species identified in clinically significant specimens in the United States. J Clin Microbiol 47(6): 1650-1656.

Alvarez, E., Stchigel A.M., et al., (2010a). «Molecular phylogenetic diversity of the emerging mucoralean fungus Apophysomyces: proposal of three new species.» Rev Iberoam Micol 27(2): 80-89.

Alvarez, E., Garcia-Hermoso D., et al., (2010b). «Molecular phylogeny and proposal of two new species of the emerging pathogenic fungus Saksenaea.» J Clin Microbiol 48(12): 4410-4416.

Alvarez, E., Cano J., et al., (2011). «Two new species of Mucor from clinical samples.» Med Mycol 49(1): 62-72.

Andresen, D., Donaldson A., et al., (2005). Multifocal cutaneous mucormycosis complicating polymicrobial wound infections in a tsunami survivor from Sri Lanka.» Lancet 365(9462): 876-878.

Baradkar, V.P. and Kumar S. (2009). Cutaneous zygomycosis due to Saksenaea vasiformis in an immunocompetent host. Indian J Dermatol 54(4): 382-384.
Barr D.J.S. (1992). Evolution and kingdoms of organisms from the perspective of a mycologist. Mycologia 84:1-11.

Benjamin, R.K. (1979). Zygomycetes and their spores, The whole fungus the sexualasexual synthesis. B. Kendrick. National Museum of Natural Science, National Museums of Canada and The Kananaskis Foundation. Ottawa, Canada, 573-616.

Benny, G.L. (1982). Zygomycetes, pp. 184-195. In S. P. Parker (Ed.). Synopsis and Classification of Living Organisms. Vol. I. McGraw-Hill Book Company, Inc., New York

Benny, G.L. (1995). "Classical morphology in zygomycete taxonomy.» Can J Bot 73(1):S725-S730.

Benny, G.L., Humber R.A. and Morton J.B. (2001). Zygomycota: Zygomycetes. Pp. 113-146. In: The Mycota VII. Systematics and Evolution. Part A. (McLaughlin, D. J., McLaughlin, E. G. and Lemke, P. A., eds.). Springer-Verlag, New York.

Berbee, M.L. and Taylor J.W. (2001). Fungal molecular evolution: gene trees and geologic time. Pp. 229-245. In: The Mycota VII. Systematics and Evolution. Part B. (McLaughlin, D. J., McLaughlin, E. G. and Lemke, P. A., eds.). Springer-Verlag, New York.

Bitar, D., Van Cauteren D., et al., (2009). «Increasing incidence of zygomycosis (mucormycosis), France, 1997-2006.» Emerg Infect Dis 15(9): 1395-1401.

Certik, M. and Shimizu S. (1999). «Biosynthesis and regulation of microbial polyunsaturated fatty acid production.» J Biosci Bioeng 87(1): 1-14.

Chakrabarti, A., Ghosh A., et al., (2003). «Apophysomyces elegans: an emerging zygomycete in India.» J Clin Microbiol 41(2): 783-788.

Chakrabarti, A., Das A., et al., (2006). «The rising trend of invasive zygomycosis in patients with uncontrolled diabetes mellitus.» Med Mycol 44(4): 335-342.

Chakrabarti, A., Chatterjee S.S., et al., (2009). «Invasive zygomycosis in India: experience in a tertiary care hospital.» Postgrad Med J 85(1009): 573-581. 
El Subphylum Mucoromycotina: generalidades y aspectos taxonómicos recientes - E. Alvarez.

Chávez, A, \& Rahal, M. (2004). Mucormicosis rinocerebral en un niño diabético: Tratamiento conservador. Rev Chil Infectol 21: 57-60.

Cruz, R, \& Piontelli, E. (2011). Enfermedad fúngica invasora en pacientes de cinco hospitales de la Región de Valparaíso, Chile: 2004 a 2009. Rev Chil Infectol 28: 123-9.

de Hoog, G.S., Guarro J., Gene J. and Figueras M.J. (2000). Atlas of clinical fungi, second addition. Centraalbureau voor Schimmelcultures, Baarn and Delft, The Netherlands.

Devi, S.C., Kanungo R., et al., (2008). «Favorable outcome of amphotericin B treatment of zygomycotic necrotizing fascitis caused by Apophysomyces elegans.» Int J Dermatol 47(4): 407-409.

England, A.C., Weinstein M., et al., (1981). «Two cases of rhinocerebral zygomycosis (mucormycosis) with common epidemiologic and environmental features.» Am Rev Respir Dis 124(4): 497-498.

Garcia-Hermoso, D., Hoinard D., Gantier J.C., Grenouillet F., Dromer F., and Dannaoui E. (2009). Molecular and phenotypic evaluation of Lichtheimia corymbifera (formerly Absidia corymbifera) complex isolates associated with human mucormycosis: rehabilitation of L. ramosa. J. Clin. Microbiol. 47: 38623870.

Gryganskyi A.P, Humber R.A., Miadlikovska J., Smith M.E., et al.,(2012). Molecular phylogeny of the Entomophthoromycota. Mol Phylogenet Evol 65(2):682-694.

Hawksworth, D.L. (1991). «The fungal dimension of biodiversity: magnitude, significance, and conservation» Mycol Res 95: 641-655.

Hawksworth, D.L. and Rossman A.Y. (1997). «Where are all the undescribed fungi?» Phytopathology 87(9): 888-891.

Hawksworth, D.L. (2001). «The magnitude of fungal diversity: the 1.5 million species estimate revisited» Mycol Res 105: 1422-1432.

Heckman, D.S., Geiser D.M., Eidell B.R., Stauffer R.L, et al., (2001). Molecular evidence for the early colonization of land by fungi and plants. Science 293: 1129-1133.
Hemashettar B.M, Patil R.N, O'Donnell K., Chaturvedi V., et al., (2011). Chronic rhinofacial mucormycosis caused by Mucor irregularis (Rhizomucor variabilis) in India. J. Clin. Microbiol. 49:2372-2375.

Hesseltine, C.W. and Ellis J.J. (1973). Mucorales. The Fungi: An Advanced Treatise. Vol. 4B: A Taxonomic Review with Keys: Basidiomycetes and Lower Fungi. G. C. Ainsworth, F. K. Sparrow, and A. S. Sussman, Academic Press, London, 187-217.

Hibbett, D.S., Binder M., Bischoff J.F., Blackwell M., et al., (2007). A higher-level phylogenetic classification of the Fungi. Mycoautores Res. 111: 509-547.

Hoffmann, K., Discher S., et al., (2007). «Revision of the genus Absidia (Mucorales, Zygomycetes) based on physiological, phylogenetic, and morphological characters; thermotolerant Absidia spp. form a coherent group, Mycocladiaceae fam. nov.» Mycol Res 111(Pt 10): 1169-1183.

Hoffmann, K., Walther G., and Voigt K. (2009). «Mycocladus vs. Lichtheimia: a correction (Lichtheimiaceae fam. nov., Mucorales, Mucoromycotina).» Mycol Res 113:277-278.

Hospenthal D.R, Chung K.K., et al., (2011). Saksenaea erythrospora infection following combat trauma. J. Clin. Microbiol. 49 (10): 3707-3709.

Humber, R.A. (2012). Entomophthoromycota: a new phylum and reclassiûcation for entomophthoroid fungi. Mycotaxon 120: 477-492.

Jain, D., Kumar Y., et al., (2006). «Zygomycotic necrotizing fasciitis in immunocompetent patients: a series of 18 cases.» Mod Pathol 19(9): 1221-1226.

James, T.Y., Kauff F., Schoch C., Matheny P.B., et al., (2006). Reconstructing the early evolution of the fungi using a six gene phylogeny. Nature 443:818822.

Kamei, K. (2001). «Animal models of zygomycosis Absidia, Rhizopus, Rhizomucor, and Cunninghamella.» Mycopathologia 152(1): 5-13.

Kaushik R, Chander J, et al., (2012). Fatal primary cutaneous zygomycosis caused by Saksenaea vasiformis: case report and review of literature. Surg Infect. 13 (2): 125-129. 
El Subphylum Mucoromycotina: generalidades y aspectos taxonómicos recientes - E. Alvarez.

Kirk, P.M., Cannon P.F., David J.C., and Stalpers J. (2001). Ainsworth and Bisby's Dictionary of the Fungi. 9th ed. CAB International, Wallingford, UK.

Kirk, P.M., Cannon P.F., et al., (2008). Ainsworth and Bisby's Dictionary of the Fungi. 10th ed. Wallingford, United Kingdom, CAB International.

Krasinski, K., Holzman R.S., et al., (1985). «Nosocomial fungal infection during hospital renovation.» Infect Control 6(7): 278-282.

Kompoti M., Michalia M., et al., (2011). Fatal cutaneous Saksenaea vasiformis infection in a critically ill trauma patient. Mycoses 54 (5): e599-601.

Kwon-Chung, K.J. and Bennett J.E. (1992). Medical Mycology. Lea \& Febiger, Philadelphia and London.

Lechevalier, P., Garcia-Hermoso D., et al., (2008). «Molecular diagnosis of Saksenaea vasiformis cutaneous infection after scorpion sting in an immunocompetent adolescent.» J Clin Microbiol 46(9): 3169-3172.

Ledgard, J.P., van Hal S., et al., (2008). «Primary cutaneous zygomycosis in a burns patient: a review.» J Burn Care Res 29(2): 286-290.

Li D.M. and Lun L.D. (2012). Mucor irregularis infection and lethal midline granuloma: a case report and review of published literature. Mycopathologia. 174:429-439.

Mayayo E., Stchigel A.M., et al. (2013). Necrotising fasciitis caused by Saksenaea vasiformis in an immunocompetent patient after a car accident. Rev Iberoam Micol 30 (1): 57-60.

Meis, J.F. and Chakrabarti A. (2009). «Changing epidemiology of an emerging infection: zygomycosis.» Clin Microbiol Infect 15 Suppl 5: 10-14.

Neblett Fanfair R., Benedict K., Bennett S., LoYiChun, et al., (2011) Fatal Fungal Soft-Tissue Infections After a Tornado - Joplin, Missouri, 2011. Morbidity and Motality Weekly Report. pp. 992.

Oddo, D, \& Acuña, G. (1988). Infecciones oportunistas en 5.612 necropsias. Santiago, Chile, 1960-1986. Bol Of Sanit Panam 22: 251-60.
O'Donnell, K., and Cigelnik E. (1994). «Phylogeny of the Zygomycota.» Abstracts of the Fifth International Mycological Congress, 14-21 Aug, Vancouver, B.C. p. 160

O'Donnell, K., Lutzoni F.M., Ward T.J. and Benny G.L. (2001). Evolutionary relationships among mucoralean fungi (Zygomycota): Evidence for family polyphyly on a large scale. Mycologia 93: 286-296.

Oliver, M.R., Van Voorhis W.C., et al., (1996). «Hepatic mucormycosis in a bone marrow transplant recipient who ingested naturopathic medicine.» Clin Infect Dis 22(3): 521-524.

Reinhardt, D.J., Licata I., et al., (1981). «Experimental cerebral zygomycosis in alloxandiabetic rabbits: variation in virulence among zygomycetes.» Sabouraudia 19(4): 245256.

Ribes, J.A., Vanover-Sams C.L., et al., (2000). «Zygomycetes in human disease.» Clin Microbiol Rev 13(2): 236-301.

Richardson, M. (2009). "The ecology of the Zygomycetes and its impact on environmental exposure.» Clin Microbiol Infect 15 Suppl 5: 2-9.

Roden, M.M., Zaoutis T.E., et al., (2005). «Epidemiology and outcome of zygomycosis: a review of 929 reported cases.» Clin Infect Dis 41(5): 634653.

Saegeman, V., Maertens J., et al., (2010). «Increasing incidence of mucormycosis in University Hospital, Belgium.» Emerg Infect Dis 16(9): 14561458.

Sandford, S.E., Josephson G.K.A., et al., (1985). «Submandibular and disseminated zygomycosis (mucormycosis) in feeder pigs.» J Am Vet Med Assoc 186:171-174.

Saravia-Flores, M., Guaran D.M., et al., (2010). «Invasive cutaneous infection caused by Apophysomyces elegans associated with a spider bite.» Mycoses 53(3): 259-261.

Schell W.A, O'Donnell K, Alspaugh J.A. (2011). Heterothallic mating in Mucor irregularis and first isolate of the species outside of Asia. Med. Mycol. 7:714-723. 
El Subphylum Mucoromycotina: generalidades y aspectos taxonómicos recientes - E. Alvarez.

Schipper, M.A.A. (1973). «A study on variability in Mucor hiemalis and related species.» Stud Mycol 4: 1-40.

Snell, B.J. and Tavakoli K. (2007). «Necrotizing fasciitis caused by Apophysomyces elegans complicating soft-tissue and pelvic injuries in a tsunami survivor from Thailand.» Plast Reconstr Surg 119(1): 448-449.

Spalloni, P, Glaser, P, Verdugo, P. (2004). Mucormicosis rinocerebral: Sobrevida en un niño con leucemia. Rev Chil Infectol 21: 53-6.

Stewardson, A.J., Holmes N.E., et al., (2009). «Cutaneous zygomycosis caused by Saksenaea vasiformis following water-related wound in a 24 -yearold immunocompetent woman.» Mycoses 52(6): 547549 .

Täger, M., Zaror, L., Martínez, P. (2012). Mucormicosis cutánea en un paciente inmuno-comprometido. Rev. Chil. Infectol 29(1): 101-107.

Taj-Aldeen S.J, Falamarzi A., et al., (2012). Rare pediatric rhino-orbital infection caused by Saksenaea vasiformis. Infection 40 (6): 703-7.

Tanabe, Y., Saikawa M., Watanabe M.M., and Sugiyama J. (2004). Molecular phylogeny of Zygomycota based on EF-1 and RPB1 sequences: limitations and utility of alternative markers to rDNA. Mol. Phylogenet. Evol. 30: 438-449.

Tanabe, Y., Watanabe M.M., et al., (2005). «Evolutionary relationships among basal fungi (Chytridiomycota and Zygomycota): Insights from molecular phylogenetics.» J Gen Appl Microbiol 51(5): 267-276.

Thompson, L., Oddo, D., Retamal, C., Ferrada, L. (1987). Orientaciones clínicas y de laboratorio en las micosis oportunistas. Rev Chil Infectol 4: 69-74.
Vega, W., Orellana M., et al., (2006). «Saksenaea vasiformis infections: case report and literature review.» Mycopathologia 162(4): 289-294.

Villarroel, F. (1985). Mucormicosis orbitaria (Ficomicosis, Zigomicosis). Arch Chil Oftalmol 42: 61-5.

Voigt, K., Cigelnik E., et al., (1999). «Phylogeny and PCR identification of clinically important Zygomycetes based on nuclear ribosomal-DNA sequence data.» J Clin Microbiol 37(12): 3957-3964.

von Arx, J.A. 1983 (1982). On Mucoraceae s. str. and other families of the Mucorales. Sydowia, 35: 10-26.

Waldorf, A.R. and Diamond R.D. (1984). «Cerebral mucormycosis in diabetic mice after intrasinus challenge.» Infect Immun 44(1): 194-195.

White, M.M., James T.Y., O'Donnell K., Cafaro M.J., et al., (2006). Phylogeny of the Zygomycota based on nuclear ribosomal sequence data. Mycologia 98: 872884.

Weitzman, I., Whittier S., et al., (1995). «Zygospores: the last word in identification of rare or atypical zygomycetes isolated from clinical specimens.» $\mathrm{J}$ Clin Microbiol 33(3): 781-783.

Wilson, P.A. (2008). «Zygomycosis due to Saksenaea vasiformis caused by a magpie peck.» Med J Aust 189(9): 521-522.

\section{CONFLICTO DE INTERESES}

El Autor declara no tener conflictos de intereses. 